\title{
Serum free thiols predict cardiovascular events and all-cause mortality in the general population: a prospective cohort study
}

Amaal E. Abdulle ${ }^{1 \dagger}$, Arno R. Bourgonje ${ }^{2 \dagger}$, Lyanne M. Kieneker ${ }^{3}$, Anne M. Koning ${ }^{4}$, S. la Bastide-van Gemert ${ }^{5}$, Marian L. C. Bulthuis ${ }^{4}$, Gerard Dijkstra ${ }^{2}$, Klaas Nico Faber ${ }^{2}$, Robin P. F. Dullaart ${ }^{6}$, Stephan J. L. Bakker ${ }^{3}$, Reinold O. B. Gans ${ }^{1}$, Ron T. Gansevoort ${ }^{3}$, Douwe J. Mulder ${ }^{1}$, Andreas Pasch ${ }^{7 \dagger}$ and Harry van Goor ${ }^{4^{*}+}$

\begin{abstract}
Background: Serum free thiols (R-SH, sulfhydryl groups) reliably reflect systemic oxidative stress. Since serum free thiols are rapidly oxidized by reactive species, systemic oxidative stress is generally associated with reduced serum free thiol levels. Free thiols associate with favorable disease outcomes in many patient cohorts, and the current hypothesis is that oxidative stress might also play an important role in cardiovascular disease. In this study, we aimed to establish the role of serum free thiols in the general population by investigating their relationship with the risk of cardiovascular (CV) events and all-cause mortality.

Methods: Participants $(n=5955)$ of the Prevention of REnal and Vascular ENd-stage Disease (PREVEND) cohort study from the general population were included. At baseline, serum levels of free thiols were quantified and adjusted to total protein levels. Protein-adjusted serum free thiol levels were studied for their associations with clinical and biochemical parameters, as well as with the risk of CV events and all-cause mortality.

Results: The mean protein-adjusted serum free thiol level was $5.05 \pm 1.02 \mu \mathrm{mol} / \mathrm{g}$ of protein. Protein-adjusted serum free thiols significantly predicted the risk of $\mathrm{CV}$ events, even after adjustment for potential confounding factors (hazard ratio [HR] per doubling 0.68 [95\% confidence interval $[\mathrm{Cl}] 0.47-1.00], P=0.048$ ). Similarly, proteinadjusted serum free thiols were significantly predictive of the risk of all-cause mortality (HR per doubling 0.66 [95\% Cl 0.44-1.00], $P=0.050$ ). Stratified analyses revealed lower HRs for subjects with a lower body mass index (BMI), without hypertension, and without diabetes. Conversely, HRs were lower in subjects with albuminuria.

\footnotetext{
* Correspondence: h.van.goor@umcg.nl

${ }^{+}$Amaal Eman Abdulle and Arno R. Bourgonje contributed equally to this manuscript.

${ }^{+}$Andreas Pasch and Harry van Goor share the last authorship.

${ }^{4}$ Department of Pathology and Medical Biology, Section Pathology,

University of Groningen - University Medical Center Groningen, Groningen, the Netherlands

Full list of author information is available at the end of the article
}

C The Author(s). 2020 Open Access This article is licensed under a Creative Commons Attribution 4.0 International License, which permits use, sharing, adaptation, distribution and reproduction in any medium or format, as long as you give appropriate credit to the original author(s) and the source, provide a link to the Creative Commons licence, and indicate if changes were made. The images or other third party material in this article are included in the article's Creative Commons licence, unless indicated otherwise in a credit line to the material. If material is not included in the article's Creative Commons licence and your intended use is not permitted by statutory regulation or exceeds the permitted use, you will need to obtain permission directly from the copyright holder. To view a copy of this licence, visit http://creativecommons.org/licenses/by/4.0/. The Creative Commons Public Domain Dedication waiver (http://creativecommons.org/publicdomain/zero/1.0/) applies to the data made available in this article, unless otherwise stated in a credit line to the data. 
(Continued from previous page)

Conclusions: In this large population-based cohort study, serum free thiols significantly predicted the risk of CV events and all-cause mortality. Our results highlight the potential significance and clinical applicability of serum free thiols since they are amendable to therapeutic intervention.

Keywords: Oxidative stress, Free thiols, Cardiovascular disease, Mortality, Population study

\section{Background}

Cardiovascular disease (CVD) is among the leading causes of morbidity and mortality globally, and during the past decade, the number of CVD-associated deaths has increased by $12.5 \%[1,2]$. Although a large proportion of the overall cardiovascular risk can be explained by known risk factors (e.g., smoking, hypertension, obesity, diabetes, and hypercholesterolemia), the pathogenesis of CVD still remains incompletely understood [3]. Current approaches to reduce the CVD burden have focused on encouraging healthier lifestyles. Other approaches have focused on the early identification of patients who are at increased risk of CVD. These latter strategies have faced multiple problems, including limited availability of non-invasive predictive biomarkers to assess the risk of cardiovascular (CV) events or mortality.

Oxidative stress is defined as the imbalance between the production of reactive oxygen species (ROS) and antioxidants. Although ROS, a collective term for oxygencontaining reactive species, are important signaling molecules regulating a variety of physiological processes, overproduction of ROS leads to cellular damage and tissue destruction. Chronic oxidative stress can easily and reliably be monitored as the depletion of serum free thiols, since they are readily oxidized by reactive species [4-6]. In support, overproduction of ROS, as reflected by reduced levels of free thiols, is frequently observed in human disease (like in chronic kidney disease, chronic heart failure, diabetes, cancer, systemic sclerosis, and Crohn's disease) that is typically associated with oxidative stress [7-10]. Free thiol groups (R-SH, sulfhydryl groups) are thought to play a protective role against oxidative stress through ROS scavenging and are an important component of the in vivo antioxidant buffer capacity. Studies from our center have demonstrated that higher levels of serum free thiols (i.e., lower levels of oxidative stress) are associated with favorable outcomes in patients with chronic heart failure [7], as well as with a beneficial outcome and cardiovascular risk profile in renal transplant recipients [11].

Oxidative stress has been acknowledged to play an important role in CVD, and conditions promoting oxidative stress have also been associated with risk of CVD [12]. Therefore, we hypothesized that serum free thiol groups, as overall markers of systemic oxidative stress, might have merit as a read-out of cardiovascular health in the general population. Moreover, free thiols might be a valuable indicator of the risk of all-cause mortality and risk of $\mathrm{CV}$ events, which could be applicable for risk stratification aimed to reduce CVD burden. Therefore, the aim of this study was to determine the predictive value of serum levels of free thiols regarding the risk of cardiovascular events and all-cause mortality in the general population.

\section{Methods}

\section{Study population}

This study used data from the Prevention of REnal and Vascular ENd-stage Disease (PREVEND) cohort study [13]. This is a large, prospective population-based cohort study with participants from the northern part of the Netherlands. The PREVEND study was set up to investigate cardiovascular and renal disease outcomes focused on albuminuria as the main risk factor. From 1997 to 1998, 85,421 inhabitants aged 28-75 years from the northern parts of the Netherlands received a questionnaire asking information about demographics, medication use, cardiovascular disease, and pregnancy, including a request to supply an early morning urine sample. Participants who had a previous diagnosis of type 1 diabetes mellitus and insulin-treated type 2 diabetes mellitus and pregnant women were excluded from the study. In total, 40,856 subjects responded to the questionnaire and were analyzed for urinary albumin concentrations. Study participants having urinary albumin concentrations $\geq 10 \mathrm{mg} / \mathrm{L}(n=6000)$ were invited to visit the outpatient research clinic of the University Medical Center Groningen (UMCG), as well as a random selection of participants with urinary albumin concentrations $<10 \mathrm{mg} / \mathrm{L} \quad(n=2592)$. Therefore, the PREVEND study consisted of an initial total of 8592 participants who completed the full study program [14]. However, serum samples from 6136 participants were collected during the second visit (2001-2003, referred to as the baseline visit in the present study). Participants who had a CV event between visit 1 and visit $2(n=181)$ were excluded from the analyses given the fact that reevents were not registered for these subjects. However, participants with a known cardiovascular disease prior to visit 2, who did not experience a CV event between visit 1 and visit 2, were included. Therefore, a total of 5955 participants were included in the current study. This study was approved by the Institutional Review Board (IRB) (full name in Dutch: "Medisch Ethische 
Toetsingscommissie" [METc]) of the UMCG. The study was conducted in accordance with the principles of the Declaration of Helsinki (2013), and all study participants provided written informed consent.

\section{Data collection}

All study participants visited the outpatient research clinic of the University Medical Center Groningen (UMCG), Groningen, the Netherlands. During the first visit, participants were requested to complete a questionnaire containing information about demographics, health status, history of cardiovascular disease (CVD), medication usage, and lifestyle habits (e.g., smoking habits), and anthropometric measurements were performed as well. At the second visit, systolic and diastolic blood pressure was automatically measured every minute for a period of $8 \mathrm{~min}$ in a supine position (Dinamap XL Model 9300 series device, Johnson \& Johnson Medical, Tampa, FL). Blood pressure was defined as the average of the last two measurements. Furthermore, serum samples were collected, and patients were asked to collect 24-h urine after they were provided with both oral and written instructions. Serum samples were stored at $-80^{\circ} \mathrm{C}$, while urine samples were stored at $-20^{\circ} \mathrm{C}$ until further analysis.

\section{Laboratory measurements}

High-sensitive C-reactive protein (hsCRP) and urinary albumin excretion (UAE) were measured by nephelometry (Dade Behring Diagnostics, Marburg, Germany). UAE was measured twice in two different 24-h urine collections, and the average was taken for analyses. Serum total cholesterol and fasting glucose were measured by dry chemistry (Eastman Kodak, Rochester, NY, USA). Serum total protein was measured using spectrophotometry (Roche Modular, Roche, Mannheim, Germany). High-density lipoprotein (HDL) cholesterol was measured using a homogeneous method (direct HDL, AerosetTM System, Abbott Laboratories, Abbott Park, IL, USA). Low-density lipoprotein (LDL) cholesterol was determined by the Friedewald formula (if triglycerides $\leq 4.5 \mathrm{mmol} / \mathrm{L}$ ). Triglycerides were measured with an enzymatic method. Serum creatinine was measured enzymatically (Roche Modular, Roche Diagnostics, Mannheim, Germany). Serum cystatin $C$ was measured using the Gentian Cystatin C Immunoassay (Gentian AS, Moss, Norway) on a modular analyzer (Roche Diagnostics). Cystatin $\mathrm{C}$ was directly calibrated using standards from the manufacturer's supplies (according to the International Federation of Clinical Chemistry Working Group for Standardization of Serum Cystatin C) [15].

\section{Serum free thiol measurements}

Measurement of serum free thiols (R-SH, sulfhydryl groups) was performed as previously described, with minor modifications $[16,17]$. After thawing, samples were 4-fold diluted using $0.1 \mathrm{M}$ Tris buffer $(\mathrm{pH}$ 8.2). Background absorption was measured at $412 \mathrm{~nm}$ using the Varioskan microplate reader (Thermo Scientific, Breda, the Netherlands), together with a reference measurement

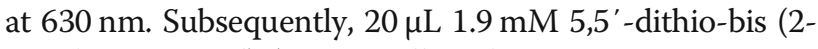
nitrobenzoic acid) (DTNB, Ellman's reagent, CAS no. 6978-3, Sigma Aldrich Corporation, St. Louis, MO, USA) was added to the samples in $0.1 \mathrm{M}$ phosphate buffer $(\mathrm{pH}$ 7.0). After an incubation time of $20 \mathrm{~min}$ at room temperature, absorbance was measured again. The final serum free thiol concentrations were established by parallel measurement of an L-cysteine (CAS no. 52-90-4, Fluka Biochemika, Buchs, Switzerland) calibration curve (range from 15.6 to $1000 \mu \mathrm{M}$ ) in $0.1 \mathrm{M}$ Tris/10 mM EDTA (pH 8.2). Intra- and interassay coefficients of variation (CV) of serum free thiol measurements were $1.9 \%$ and $5.0 \%$, respectively. Finally, serum free thiol concentrations were adjusted to total serum protein (measured according to standard procedures) by calculating the free thiol/total protein ratio $(\mu \mathrm{mol} / \mathrm{g}$ of protein). This adjustment was performed since serum proteins harbor the largest amount of free thiols and therefore greatly determine the quantity of potentially detectable free thiol groups [18].

\section{Study outcomes and definitions}

Estimated glomerular filtration rates (eGFR) were calculated using the combined creatinine cystatin C-based Chronic Kidney Disease Epidemiology Collaboration (CKD-EPI) equation [19]. Type 2 diabetes mellitus (T2DM) was defined as a fasting glucose level $\geq 7.0 \mathrm{mM}$ or the use of antidiabetic medications according to the guidelines of the American Diabetic Association (ADA). Hypercholesterolemia was defined as a serum total cholesterol level $>6.5 \mathrm{mmol} / \mathrm{L}$, serum $\mathrm{HDL}$ cholesterol level $<0.9 \mathrm{mmol} / \mathrm{L}$, or the use of lipid-lowering drugs at baseline. Fatal and non-fatal cardiovascular events (CV) (i.e., combined outcomes of acute myocardial infarction (AMI), acute or subacute ischemic heart disease (IHD), coronary artery bypass grafting (CABG), percutaneous transluminal coronary angioplasty (PTCA), intracerebral hemorrhage, other intracranial hemorrhages, subarachnoid hemorrhage, occlusion and stenosis of precerebral or cerebral arteries, and other vascular interventions such as carotid desobstruction, aorta peripheral bypass surgery, or percutaneous transluminal femoral angioplasty) and all-cause mortality were considered primary endpoints of the current study. These outcomes were determined by using information from the Dutch National Registry of all hospital discharge diagnoses (Prismant). This information was classified in accordance with the International Statistical Classification of Diseases (ICD-10) and the International Classification of Health Interventions [20]. In addition, the classical 3-point major 
adverse cardiovascular events (3P-MACE) was considered as the composite endpoint for $\mathrm{CV}$ events, consisting of fatal CV events, non-fatal myocardial infarction, and nonfatal stroke [21].

\section{Statistical analysis}

Data analysis was performed using the SPSS Statistics 25.0 software package (SPSS Inc., Chicago, IL, USA), Stata 15.1 (Stata Corp LLC, College Station, TX, USA), and $\mathrm{R}$ version 3.5.2. (Vienna, Austria). Data visualization was performed using GraphPad Prism 5.0 (GraphPad Software, San Diego, CA, USA) and RStudio (version
1.2.1335; RStudio, Boston, MA). Demographic and clinical characteristics were presented as mean \pm standard deviation (SD), median (interquartile range [IQR]) in case of skewed variables, or proportions $n$ with corresponding percentages (\%). Normality was assessed and visualized using histograms and normal probability plots (Q-Q plots). Between-group comparisons for continuous variables were performed using one-way analysis of variance (ANOVA) or Kruskal-Wallis tests, while for nominal variables, chi-square tests were performed, as appropriate. Protein-adjusted serum free thiol concentrations were ${ }^{2} \log$-transformed to facilitate result

Table 1 Demographic and clinical characteristics of the study population

\begin{tabular}{|c|c|c|c|c|c|}
\hline & Total, $N=5955$ & $\mathrm{~T} 1, N=1985$ & $\mathrm{~T} 2, N=1985$ & $\mathrm{~T} 3, N=1985$ & $P$ value \\
\hline Serum free thiol ( $\mu \mathrm{mol} / \mathrm{g}$ ) levels (adjusted for protein) & $5.05( \pm 1.02) \mu \mathrm{mol} / \mathrm{g}$ & $<4.65 \mu \mathrm{mol} / \mathrm{g}$ & $4.65-5.46 \mu \mathrm{mol} / \mathrm{g}$ & $>5.46 \mu \mathrm{mol} / \mathrm{g}$ & \\
\hline Age (years) & $51.6(43.3-61.7)$ & $57.6(47.8-66.6)$ & $51.1(43.4-59.8)$ & $47.7(41.1-55.3)$ & $<0.001$ \\
\hline Female, $n(\%)$ & $3038(51.0 \%)$ & $1084(54.6 \%)$ & $1013(51.0 \%)$ & $941(47.4 \%)$ & $<0.001$ \\
\hline \multicolumn{6}{|l|}{ Blood pressure } \\
\hline $\mathrm{SBP}(\mathrm{mmHg})$ & $126( \pm 19)$ & $129( \pm 20)$ & $125( \pm 18)$ & $123( \pm 17)$ & $<0.001$ \\
\hline $\mathrm{DBP}(\mathrm{mmHg})$ & $73( \pm 9)$ & $74( \pm 9)$ & $73( \pm 9)$ & $73( \pm 9)$ & $<0.001$ \\
\hline Heart rate (bpm) & $68( \pm 10)$ & $69( \pm 11)$ & $69( \pm 10)$ & $68( \pm 10)$ & 0.44 \\
\hline \multicolumn{6}{|l|}{ Obesity variables } \\
\hline BMI $\left(\mathrm{kg} / \mathrm{m}^{2}\right)$ & $26.0(23.6-28.9)$ & $26.8(24.3-29.9)$ & $26.0(23.6-28.7)$ & $25.3(23.0-28.0)$ & $<0.001$ \\
\hline Waist circumference $(\mathrm{cm})$ & $91.0(82.0-100.0)$ & $94.0(85.0-102.0)$ & $91.0(82.0-100.0)$ & $89.0(81.0-98.0)$ & $<0.001$ \\
\hline Smoking, $n(\%)$ & & & & & $<0.001$ \\
\hline Never, $n(\%)$ & $1757(29.5 \%)$ & $583(29.4 \%)$ & $578(29.1 \%)$ & $596(30.0 \%)$ & \\
\hline Current, $n(\%)$ & $1655(27.8 \%)$ & $494(24.9 \%)$ & $538(27.1 \%)$ & $623(31.4 \%)$ & \\
\hline Former, $n(\%)$ & $2472(41.5 \%)$ & $887(44.7 \%)$ & $848(42.7 \%)$ & 737 (37.1\%) & \\
\hline History of cardiovascular disease, $n(\%)$ & 217 (3.6\%) & $100(5.0 \%)$ & $69(3.5 \%)$ & $48(2.4 \%)$ & $<0.001$ \\
\hline Diabetes, $n(\%)$ & $144(2.4 \%)$ & $76(3.8 \%)$ & $34(1.7 \%)$ & $34(1.7 \%)$ & $<0.001$ \\
\hline \multicolumn{6}{|l|}{ Medication, $n$ (\%) } \\
\hline Antihypertensive drugs, $n$ (\%) & $1037(17.4 \%)$ & $481(24.2 \%)$ & $307(15.5 \%)$ & $249(12.5 \%)$ & $<0.001$ \\
\hline Lipid-lowering drugs, $n$ (\%) & $371(6.2 \%)$ & $155(7.8 \%)$ & $126(6.3 \%)$ & $90(4.5 \%)$ & $<0.001$ \\
\hline Antidiabetic drugs, $n$ (\%) & $78(1.3 \%)$ & $40(2.0 \%)$ & 19 (1.0\%) & $19(1.0 \%)$ & 0.006 \\
\hline \multicolumn{6}{|l|}{ Laboratory measurements } \\
\hline Total cholesterol (mmol/L) & $5.5( \pm 1.0)$ & $5.5( \pm 1.0)$ & $5.5( \pm 1.0)$ & $5.4( \pm 1.1)$ & $<0.001$ \\
\hline $\mathrm{HDL}(\mathrm{mmol} / \mathrm{L})$ & $1.2(1.0-1.5)$ & $1.2(1.1-1.5)$ & $1.2(1.0-1.4)$ & $1.2(1.0-1.4)$ & 0.68 \\
\hline $\mathrm{LDL}(\mathrm{mmol} / \mathrm{L})$ & $3.4(2.7-4.2)$ & $3.5(2.8-4.2)$ & $3.3(2.7-4.1)$ & $3.3(2.6-4.1)$ & 0.20 \\
\hline Triglycerides (mmol/L) & $1.1(0.8-1.6)$ & $1.1(0.8-1.6)$ & $1.1(0.8-1.6)$ & $1.0(0.8-1.6)$ & 0.001 \\
\hline hsCRP (mg/L) & $1.3(0.6-2.9)$ & $1.6(0.8-3.9)$ & $1.3(0.6-2.8)$ & $1.0(0.5-2.3)$ & $<0.001$ \\
\hline Fasting glucose (mmol/L) & $4.7(4.4-5.2)$ & $4.8(4.5-5.3)$ & $4.7(4.4-5.2)$ & $4.7(4.4-5.2)$ & $<0.001$ \\
\hline eGFR (mL/min/1.73 m²) & $94.5(82.2-104.7)$ & $86.5(74.7-99.0)$ & $95.0(83.9-14.0)$ & $99.8(90.1-108.2)$ & $<0.001$ \\
\hline Albuminuria (mg/L) & $8.5(6.0-15.0)$ & $9.3(6.2-19.0)$ & $8.4(6.0-14.0)$ & $8.1(5.95-13.1)$ & $<0.001$ \\
\hline CV events, $n(\%)$ & $402(6.8 \%)$ & $184(9.3 \%)$ & $127(6.4 \%)$ & $91(4.6 \%)$ & $<0.001$ \\
\hline Mortality, n (\%) & $316(5.3 \%)$ & $171(8.6 \%)$ & $85(4.3 \%)$ & $60(3.0 \%)$ & $<0.001$ \\
\hline
\end{tabular}

Data are presented as mean \pm SD, proportions $n$ with corresponding percentages (\%) or median (IQR)

$P$ values in bold indicate statistical significance

$S B P$ systolic blood pressure, $D B P$ diastolic blood pressure, $B M I$ body mass index, $H D L$ high-density lipoprotein, $L D L$ low-density lipoprotein, $h s C R P$ high-sensitive $C$ reactive protein, eGFR estimated glomerular filtration rate, $C V$ cardiovascular 
interpretation (per doubling). Survival distributions were assessed for tertiles of protein-adjusted serum free thiol concentrations using Kaplan-Meier curves and compared with log-rank tests. Survival time was defined from baseline (time of serum sample collection) until the date of the last examination that participants attended: the incidence of the cardiovascular (CV) event, death, or January 1, 2010 (end of follow-up). Cox proportional hazards regression analyses were performed to assess the association between protein-adjusted serum free thiols and the risk of $\mathrm{CV}$ events or all-cause mortality. The results were expressed as hazard ratios (HRs) with corresponding 95\% confidence intervals. Univariable associations were followed by multivariable Cox regression models to adjust for potential confounding factors and stratified analyses to assess HRs across several subgroups. To evaluate the discriminative capacity of Cox regression models, receiver operating characteristics (ROC) analyses were performed by calculating the Harrell $\mathrm{C}$ index for the various models. The Harrell $\mathrm{C}$ index represents the area under the ROC curve (AUC) and is compatible with time-to-event data. Finally, Cox regression analyses were repeated with restricted cubic splines with three knots to evaluate potential nonlinearity of associations between protein-adjusted serum-free thiols and CV events or all-cause mortality. Non-linearity was evaluated by likelihood ratio tests, where nested models were compared using linear or linear and cubic spline terms. In addition, a sensitivity analysis was performed in which oversampling of subjects with higher urinary albumin concentrations was addressed by using design-based Cox proportional hazards regression models. Two-tailed $P$ values $\leq 0.05$ were considered statistically significant.

\section{Selection of confounding variables}

In line with the guidelines for adjusting for confounders as described previously, we used causal models (directed acyclic graphs (DAGs) and their associated theory) to distinguish the appropriate set of confounders for estimating our effect of interest [22-24]. Based on previously established theory in our field and respecting constraints imposed by time and logic, the DAG represents the causal mechanisms we hypothesize to be underlying the variables at hand (scenario 1) (Additional file 1: Figure S1) [9, 25-31].

Arrows depict hypothesized causal (direct) effects between variables, whereas the absence of an arrow between two variables represents the assumption of no such direct effect. Focusing on the effect of oxidative stress on the outcome (cardiovascular events and allcause mortality), we can now identify those variables for which conditioning in the analysis is necessary in order to obtain an unconfounded effect estimate in our statistical analysis. This is performed by determining unblocked causal paths in the graph from oxidative stress to outcome, which-when left unblocked-would bias the desired effect estimate. Consequently, we concluded that conditioning on the variables gender, age, smoking, systolic blood pressure, total cholesterol, diabetes, and hsCRP (as a proxy for inflammation) in the analysis would provide an unconfounded effect estimate of oxidative stress on the outcome.

As there could also be arguments that would lead the arrow pointing from hsCRP (as a proxy for inflammation) to oxidative stress to point the other way around [32], we additionally included BMI in our set of confounders to eliminate additional potential bias introduced by that scenario (scenario 2). It has to be noted that including BMI in the DAG (scenario 1) should causally have no influence on the effect estimation (Additional file 1: Figure S1).

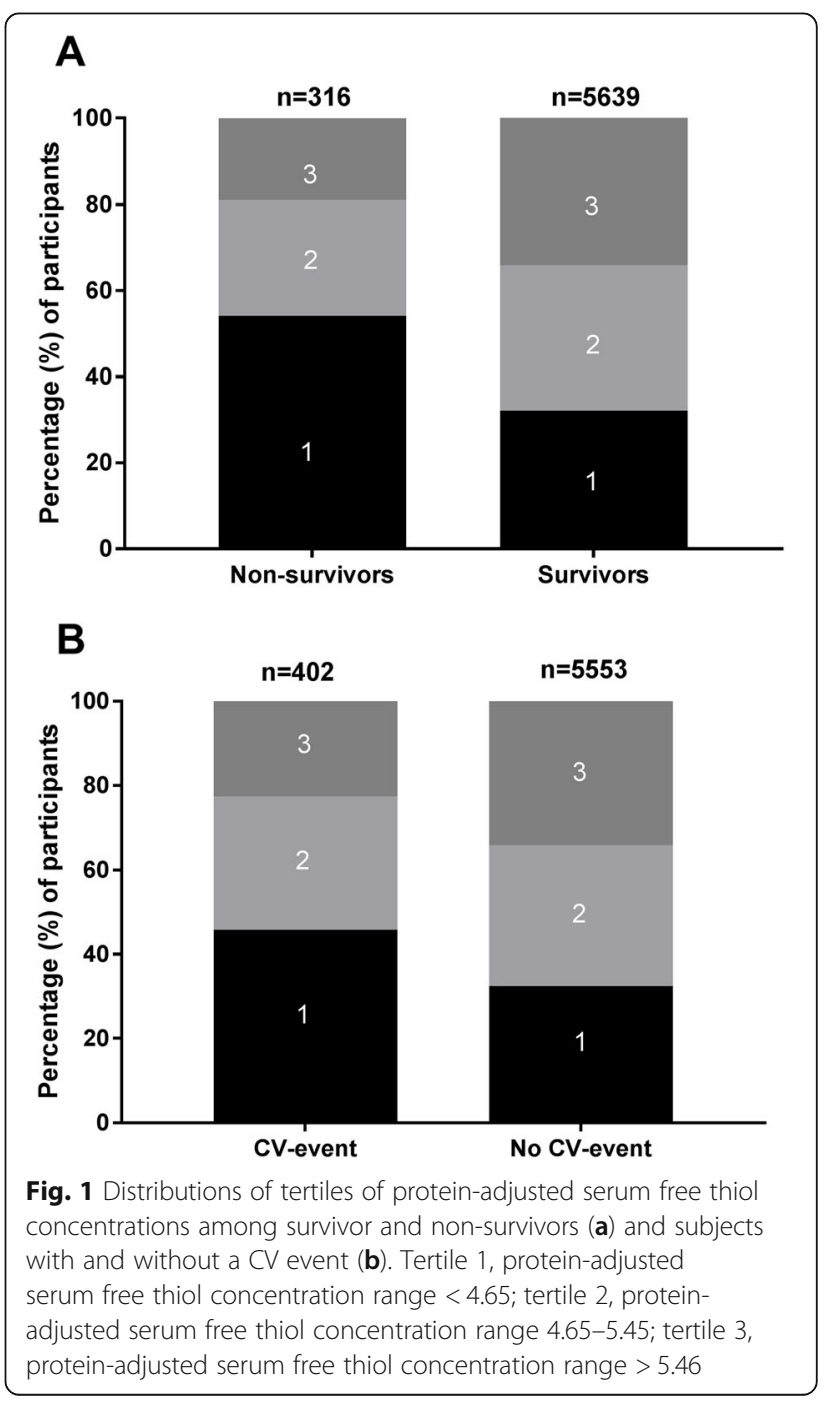




\section{Results}

\section{Patient characteristics}

The baseline characteristics of the study population are presented in Table 1. The mean age was 51.6 (43.361.7 ) years, and $51 \%$ of the participants were female. Participants in the lowest tertile of protein-adjusted serum free thiols were significantly older and had a higher body mass index (BMI) as compared to the other groups (both $P<0.001$ ). A history of cardiovascular disease was observed in $3.6 \%$ of the total cohort, and diabetes was found in $2.4 \%$ of the total number of participants. Mean protein-adjusted serum free thiol level was $5.05 \pm 1.02 \mu \mathrm{mol} / \mathrm{g}$ of protein (median $5.07 \mu \mathrm{mol} / \mathrm{g}$ of protein, full range $1.47-9.33 \mu \mathrm{mol} / \mathrm{g}$ ). Cardiovascular diseases and diabetes were more frequently observed in subjects within the lowest free thiol tertile (both $P<0.001$ ). Higher rates of mortality $(P<0.001)$ and cardiovascular events $(P<0.001)$ were more often observed in subjects within the lowest tertile of serum free thiols. Figure 1 shows the distribution of serum free thiol tertiles among survivors and nonsurvivors and among subjects with and without a CV event.

\section{Protein-adjusted serum free thiols and risk of} cardiovascular events and all-cause mortality

The mean follow-up of study participants was $7.7 \pm 2.0$ years, during which 402 (6.8\%) cardiovascular events occurred. The highest frequency of cardiovascular events was observed in participants within the lowest tertile of proteinadjusted serum free thiols (184 [9.3\%], $P<0.001)$. Kaplan-
Meier survival analysis showed a significant difference in the survival distribution between tertiles of protein-adjusted serum free thiols among participants with and without cardiovascular events (Fig. 2a, $P<0.0001$, log-rank test). Cox proportional hazards regression analyses and corresponding Harrell $\mathrm{C}$ indices demonstrated a statistically significant prediction of the risk of cardiovascular events (Table 2 (A), model 1, hazard ratio [HR] per doubling of concentration 0.39 [0.29-0.51], $P<0.001)$. After adjustment for potential confounding factors (age, sex, systolic blood pressure, BMI, total cholesterol, diabetes, current smoking, and hsCRP levels), this predictive association remained statistically significant (Table 2 (A), model 3, HR per doubling of concentration 0.68 [0.47-1.00], $P=0.048)$. These results remained unchanged when the 3P-MACE was considered as the composite endpoint. Discriminative capacity of Cox regression models significantly improved after adjustment for potential confounding factors (Fig. 3a-c).

During follow-up, 316 (5.3\%) subjects died. The highest mortality rate occurred in the lowest tertile of proteinadjusted serum-free thiols (171 [8.6\%], $P<0.001)$. KaplanMeier survival analysis showed a significantly differential survival distribution between tertiles of protein-adjusted serum free thiols among survivors and non-survivors (Fig. 2b, $P<0.0001$, log-rank test). Cox proportional hazards regression analyses and corresponding Harrell $\mathrm{C}$ indices showed a significant inverse predictive association between protein-adjusted serum free thiol concentrations and the risk of all-cause mortality (Table 2 (B), model 1, HR per doubling of concentration 0.30 [0.22-0.41], $P<$ 0.001). This association remained significant after

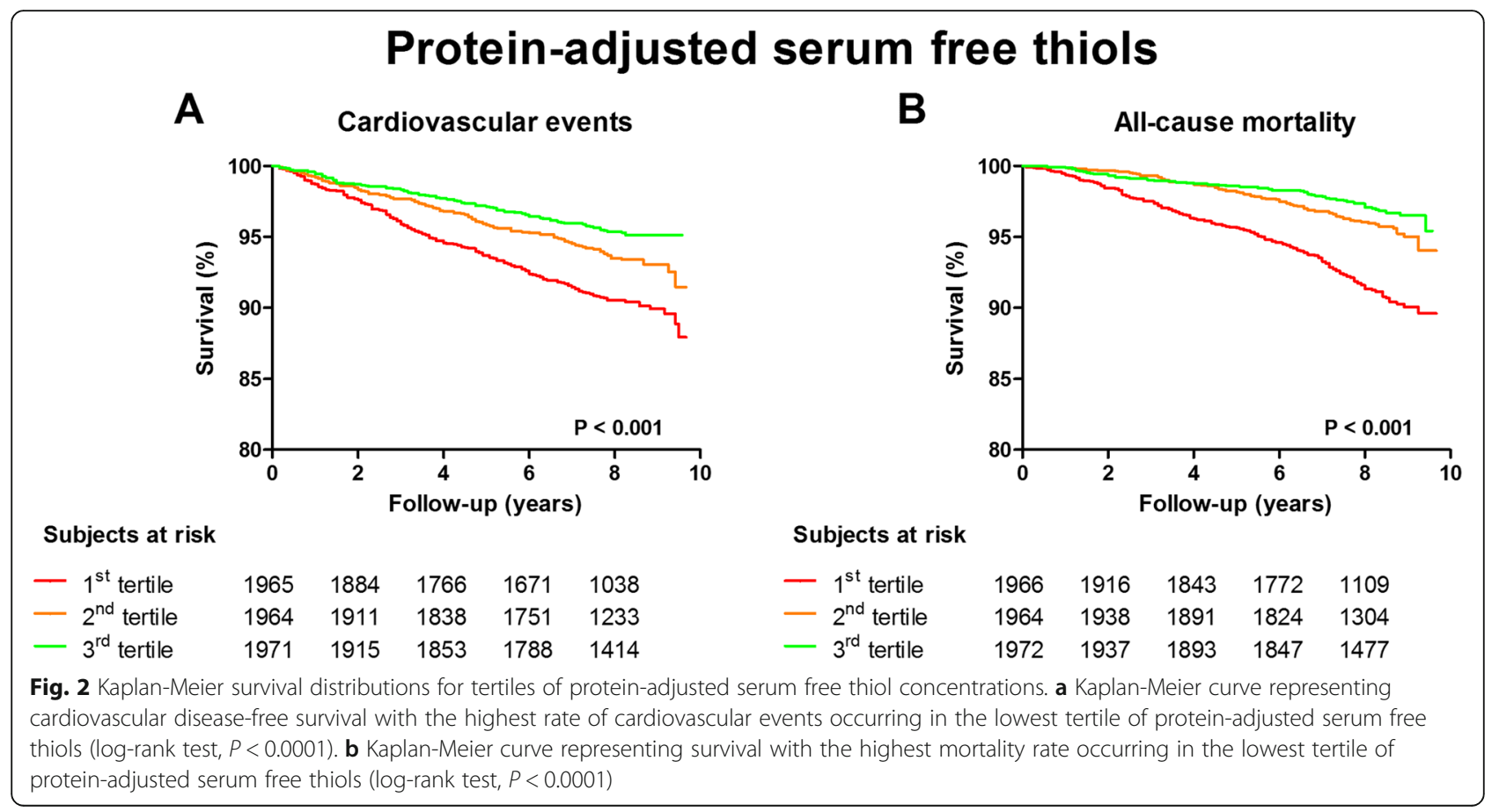


Table 2 Cox proportional hazards regression models of the association between protein-adjusted serum-free thiols and potential confounding factors with (A) cardiovascular events and (B) all-cause mortality

\begin{tabular}{|c|c|c|c|c|}
\hline & \multirow[t]{2}{*}{ HR per doubling } & \multicolumn{3}{|c|}{ Tertiles of protein-adjusted serum-free thiols } \\
\hline & & $<4.65 \mu \mathrm{mol} / \mathrm{g}$ & $4.65-5.46 \mu \mathrm{mol} / \mathrm{g}$ & $>5.46 \mu \mathrm{mol} / \mathrm{g}$ \\
\hline \multicolumn{5}{|c|}{ A. Cardiovascular events } \\
\hline Model 1 & $0.39(0.29-0.51), P<0.001$ & 1.00 (reference) & $0.66(0.53-0.83), P<0.001$ & $0.47(0.37-0.61), P<\mathbf{0 . 0 0 1}$ \\
\hline Model 2 & $0.71(0.51-0.97), P=0.031$ & 1.00 (reference) & $0.94(0.75-1.19), P=0.600$ & $0.82(0.63-1.07), P=0.135$ \\
\hline Model 3 & $0.68(0.47-1.00), \boldsymbol{P}=\mathbf{0 . 0 4 8}$ & 1.00 (reference) & $1.00(0.77-1.30), P=0.998$ & $0.81(0.59-1.10), P=0.178$ \\
\hline \multicolumn{5}{|c|}{ B. All-cause mortality } \\
\hline Model 1 & $0.30(0.22-0.41), P<0.001$ & 1.00 (reference) & $0.47(0.37-0.61), P<0.001$ & $0.34(0.25-0.45), \boldsymbol{P}<\mathbf{0 . 0 0 1}$ \\
\hline Model 2 & $0.71(0.50-1.01), P=0.056$ & 1.00 (reference) & $0.77(0.59-1.00), \mathbf{P}=\mathbf{0 . 0 4 8}$ & $0.73(0.54-1.00), \boldsymbol{P}=\mathbf{0 . 0 4 9}$ \\
\hline Model 3 & $0.66(0.44-1.00), P=\mathbf{0 . 0 5 0}$ & 1.00 (reference) & $0.87(0.65-1.16), P=0.337$ & $0.68(0.47-0.98), \boldsymbol{P}=\mathbf{0 . 0 3 7}$ \\
\hline
\end{tabular}

Model 1: crude; model 2: model 1, age- and sex-adjusted; model 3 (based on DAG): model 2, hsCRP, current smoking, systolic blood pressure, total cholesterol, diabetes, and BMI

$P$ values in bold indicate statistical significance

$H R$ hazard ratio

adjustment for potential confounders (Table 2 (B), model 3, 0.66 [0.44-1.00], $P=0.050)$. Discriminative capacity of Cox regression models significantly improved after adjustment for potential confounding factors (Fig. 3d-f). Restricted cubic splines showed no significant deviances from linear associations with either the incidence of CV events or allcause mortality for protein-adjusted serum free thiol concentrations (Additional file 2: Figure S2A-B, $P=0.258$ and $P=0.642$, respectively). In weighted analyses, in which we accounted for the oversampling of subjects with higher urinary albumin concentrations, the results (free thiols per doubling) differed slightly (CV events, 0.65 [95\% CI $0.38-$ 1.10]; all-cause mortality, 0.59 [95\% CI 0.34-1.02]).

\section{Stratified analyses}

Stratified analyses for the association between proteinadjusted serum free thiols and the risk of CV events showed consistently inverse associations in various subgroups, except for associations stratified by the presence of hypertension $\left(P_{\text {interaction }}<0.001\right)$ (Table 3; Fig. 4 ).

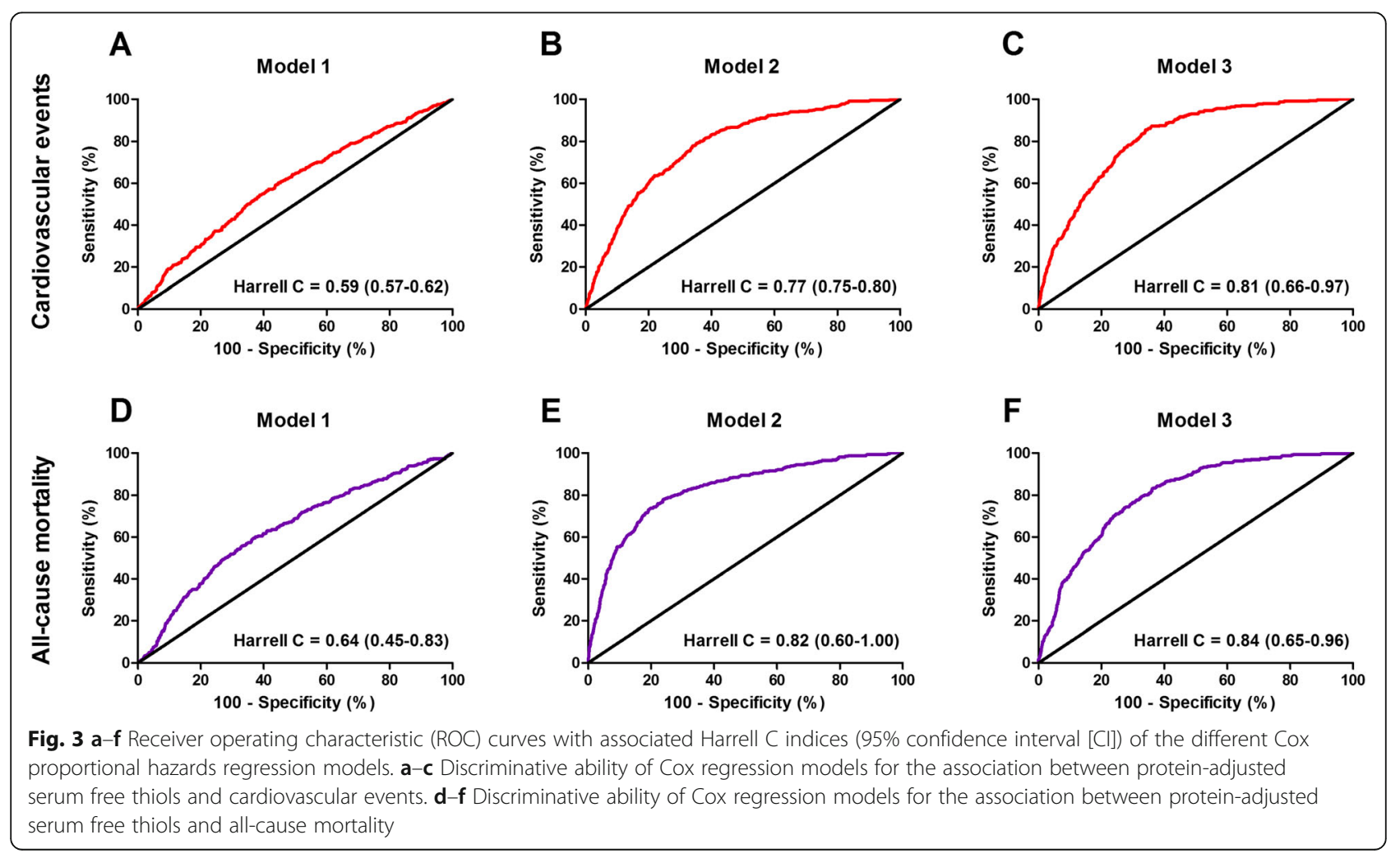


Stratification by BMI and the presence of albuminuria, hypertension, and diabetes showed significant differences between groups. Corresponding HRs were lower for subjects with a lower BMI, without hypertension, and without diabetes. Conversely, HRs were lower in subjects with albuminuria.

In subjects with a known history of CVD $(n=217$, $3.6 \%$ ), Cox proportional hazards regression analyses revealed no significant age- and sex-adjusted associations

Table 3 Stratified analyses for the association between proteinadjusted serum free thiols and the risk of cardiovascular (CV) events across various subgroups

\begin{tabular}{|c|c|c|c|c|c|}
\hline Variable & CV events $(n)$ & Total $(n)$ & $\mathrm{HR}^{*}$ & $95 \% \mathrm{Cl}$ & $P_{\text {interaction }}$ \\
\hline Overall & 402 & 5955 & 0.68 & $0.47-1.00$ & 0.048 \\
\hline \multicolumn{6}{|l|}{ Gender } \\
\hline Female & 87 & 2497 & 0.87 & $0.42-1.77$ & \multirow[t]{2}{*}{0.976} \\
\hline Male & 221 & 2180 & 0.58 & $0.38-0.90$ & \\
\hline \multicolumn{6}{|l|}{ BMI } \\
\hline$<25.0$ & 68 & 1910 & 0.53 & $0.23-1.21$ & \multirow[t]{2}{*}{$<0.001$} \\
\hline$>25.0$ & 239 & 2762 & 0.71 & $0.47-1.08$ & \\
\hline \multicolumn{6}{|c|}{ Albuminuria } \\
\hline No & 237 & 4285 & 0.71 & $0.46-1.08$ & \multirow[t]{2}{*}{0.008} \\
\hline Yes & 71 & 394 & 0.52 & $0.24-1.12$ & \\
\hline \multicolumn{6}{|c|}{ Hypertension } \\
\hline No & 219 & 4143 & 0.55 & $0.36-0.86$ & \multirow[t]{2}{*}{$<0.001$} \\
\hline Yes & 94 & 501 & 1.06 & $0.51-2.19$ & \\
\hline \multicolumn{6}{|c|}{ CVD history } \\
\hline No & 271 & 4526 & 0.69 & $0.46-1.04$ & \multirow[t]{2}{*}{0.327} \\
\hline Yes & 37 & 155 & 0.47 & $0.18-1.21$ & \\
\hline \multicolumn{6}{|l|}{ Diabetes } \\
\hline No & 289 & 4592 & 0.67 & $0.45-0.98$ & \multirow[t]{2}{*}{0.012} \\
\hline Yes & 15 & 73 & 0.79 & $0.16-3.83$ & \\
\hline \multicolumn{6}{|l|}{ Smoking } \\
\hline No & 200 & 3402 & 0.78 & $0.49-1.27$ & \multirow[t]{2}{*}{0.484} \\
\hline Yes & 108 & 1279 & 0.46 & $0.26-0.83$ & \\
\hline \multicolumn{6}{|c|}{ Alcohol consumption } \\
\hline No & 99 & 1134 & 0.72 & $0.38-1.36$ & \multirow[t]{2}{*}{0.911} \\
\hline Yes & 209 & 3547 & 0.63 & $0.40-0.98$ & \\
\hline \multicolumn{6}{|c|}{ Hypercholesterolemia } \\
\hline No & 260 & 4372 & 0.61 & $0.41-0.90$ & \multirow[t]{2}{*}{0.062} \\
\hline Yes & 41 & 242 & 0.83 & $0.28-2.42$ & \\
\hline
\end{tabular}

Stratifications by BMI, albuminuria, hypertension, diabetes, and hypercholesterolemia showed significant differences between groups. Corresponding hazard ratios (HRs) are consistently lower for subjects with a more favorable cardiovascular risk profile (i.e., lower BMI and the absence of hypertension and diabetes), whereas the presence of CVD history or albuminuria shows lower HRs as well

$C V$ cardiovascular, $H R$ hazard ratio, $C l$ confidence interval, $B M I$ body mass index, CVD cardiovascular disease

*Adjusted for potential confounding factors (sex, age, hsCRP, current smoking, systolic blood pressure, total cholesterol, diabetes, and BMI) between protein-adjusted serum free thiol concentrations and the risk of $\mathrm{CV}$ events (Table 4 (A), HR per doubling of concentration 0.52 [0.23-1.19], $P=0.120$ ). However, we observed significant age- and sex-adjusted associations in this subgroup between protein-adjusted serum free thiol concentrations and the risk of all-cause mortality when comparing the upper two tertiles with the lowest tertile (Table 4 (B), HR per doubling of concentration: tertile 2, 0.48 [0.24-0.96], $P=0.038$; tertile 3, 0.40 [0.16-0.99], $P=0.047$ ). Although there was a large difference in the sample size between subgroups of subjects with and without a history of CVD $(n=217,3.6 \%$ vs. $n=5738,96.4 \%$, respectively), the discriminative capacity of these models (Harrell $\mathrm{C}$ indices) was higher in subjects without a known history of CVD compared to subjects with a history of CVD (Fig. 5).

\section{Discussion}

In this large population-based cohort, we found that protein-adjusted serum free thiol concentrations were able to significantly predict the risk of $\mathrm{CV}$ events and all-cause mortality. After adjustment for confounders, this predictive capacity of protein-adjusted serum free thiol concentrations (per doubling) regarding the risk of $\mathrm{CV}$ events, as well as the risk of all-cause mortality, remained statistically significant. Collectively, our results shed light on the potential significance of serum levels of free thiols for risk stratification in primary and secondary prevention settings to reduce CVD burden in the general population.

Over the last couple of decades, increasing attention has been paid to the role of oxidative stress in CVD, since it may lead to endothelial dysfunction [33]. For instance, it was previously shown that thiols are an oxidative stress marker in subjects with an acute myocardial infarction [34] and that plasma thiols are significantly lower in subjects with congestive heart failure, as compared to healthy subjects [35]. In the current study, we tested the hypothesis that protein-adjusted serum free thiols, due to the ROS-scavenging properties of free thiols, are markers of the systemic redox status that might be associated with the risk of $\mathrm{CV}$ events and mortality in the general population. Indeed, our results show that protein-adjusted serum free thiol concentrations (per doubling) are inversely associated with the risk of $\mathrm{CV}$ events after adjustment for potential confounders. Regarding mortality, after correcting for potential confounders, we observed a significant association with protein-adjusted serum free thiols. This postulation is in line with previous findings showing that serum thiols are independently associated with $\mathrm{CV}$ risk scores at the population level [36]. As compared to this crosssectional study, however, the current study is much larger and of longitudinal origin (10 years of follow-up) and 


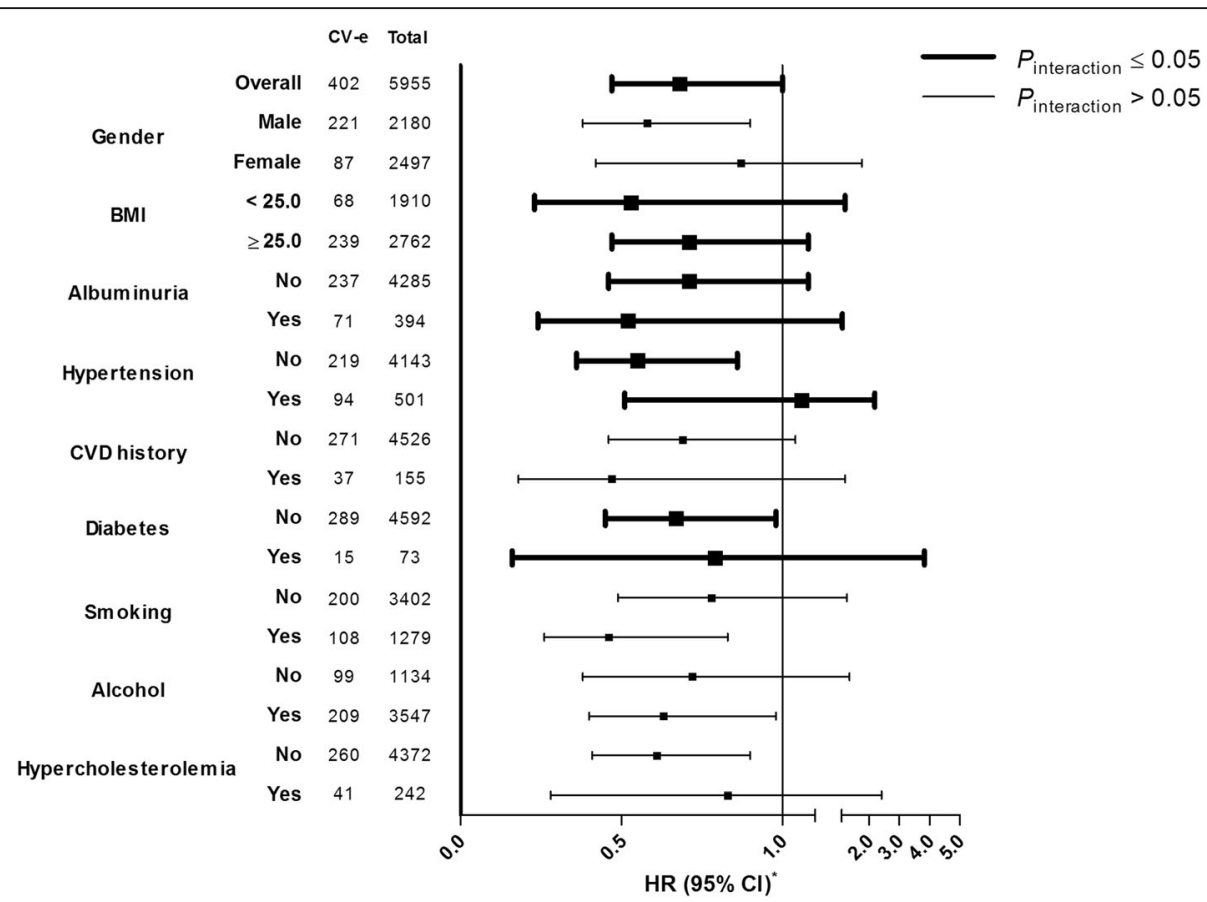

Fig. 4 Associations between protein-adjusted serum free thiol levels and the risk of cardiovascular (CV) events across various subgroups. Hazard ratios (HRs) are shown with corresponding 95\% confidence intervals (CI). HRs represent consistently inverse associations across subgroups, with the exception of stratification by the presence of hypertension $\left(P_{\text {interaction }}<0.001\right)$. Stratifications by BMI, albuminuria, hypertension, diabetes, and hypercholesterolemia show significant interactions. Corresponding HRs are consistently lower for subjects with a more favorable cardiovascular risk profile (i.e., lower BMI and the absence of hypertension and diabetes), whereas the presence of CVD history or albuminuria shows lower HRs as well. *HRs adjusted for potential confounding factors (sex, age, hsCRP, current smoking, systolic blood pressure, total cholesterol, diabetes, and BMI). CV-e, cardiovascular events; BMI, body mass index; eGFR, estimated glomerular filtration rate; CVD, cardiovascular disease; $\mathrm{HR}$, hazard ratio; Cl, confidence interval

demonstrates associations of free thiols with the occurrence of $\mathrm{CV}$ events and all-cause mortality.

In our stratified analyses, the association between protein-adjusted serum free thiols and the risk of $\mathrm{CV}$ events showed no significant interaction between subjects with and without CVD. Moreover, we demonstrated that the association between protein-adjusted serum free thiols and the risk of CV events was consistently inversely associated in various subgroups, except for the association stratified by the presence of hypertension. Free thiols were

Table 4 Cox proportional hazards regression models of the age- and sex-adjusted associations between protein-adjusted serum free thiols and the risk of (A) cardiovascular events and (B) all-cause mortality in participants with and without a history of cardiovascular disease (CVD)

\begin{tabular}{|c|c|c|c|c|}
\hline & \multirow[t]{2}{*}{ HR per doubling } & \multicolumn{3}{|c|}{ Tertiles of protein-adjusted serum-free thiols } \\
\hline & & $<4.65 \mu \mathrm{mol} / \mathrm{g}$ & $4.65-5.46 \mu \mathrm{mol} / \mathrm{g}$ & $>5.46 \mu \mathrm{mol} / \mathrm{g}$ \\
\hline \multicolumn{5}{|c|}{ A. Cardiovascular events } \\
\hline Total cohort & $0.71(0.51-0.97), P=\mathbf{0 . 0 3 1}$ & 1.00 (reference) & $0.94(0.75-1.19), P=0.600$ & $0.82(0.63-1.07), P=0.135$ \\
\hline No history of CVD & $0.77(0.54-1.08), P=0.127$ & 1.00 (reference) & $0.96(0.75-1.23), P=0.761$ & $0.83(0.62-1.09), P=0.181$ \\
\hline History of CVD & $0.52(0.23-1.19), P=0.120$ & 1.00 (reference) & $0.82(0.42-1.61), P=0.567$ & $0.84(0.38-1.86), P=0.670$ \\
\hline \multicolumn{5}{|l|}{ B. All-cause mortality } \\
\hline Total cohort & $0.71(0.50-1.01), P=0.056$ & 1.00 (reference) & $0.77(0.59-1.00), \mathbf{P}=\mathbf{0 . 0 4 8}$ & $0.73(0.54-1.00), \boldsymbol{P}=\mathbf{0 . 0 4 9}$ \\
\hline No history of CVD & $0.80(0.54-1.18), P=0.253$ & 1.00 (reference) & $0.81(0.61-1.09), P=0.159$ & $0.81(0.58-1.12), P=0.206$ \\
\hline History of CVD & $0.47(0.22-1.02), P=0.055$ & 1.00 (reference) & $0.48(0.24-0.96), P=\mathbf{0 . 0 3 8}$ & $0.40(0.16-0.99), \boldsymbol{P}=\mathbf{0 . 0 4 7}$ \\
\hline
\end{tabular}

All models were age- and sex-adjusted

$P$ values in bold indicate statistical significance

CVD cardiovascular disease, $H R$ hazard ratio 
A

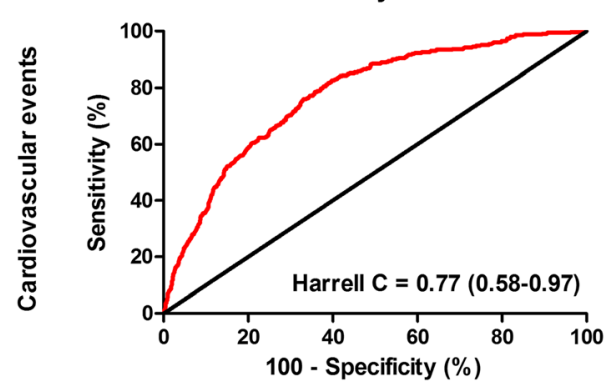

C

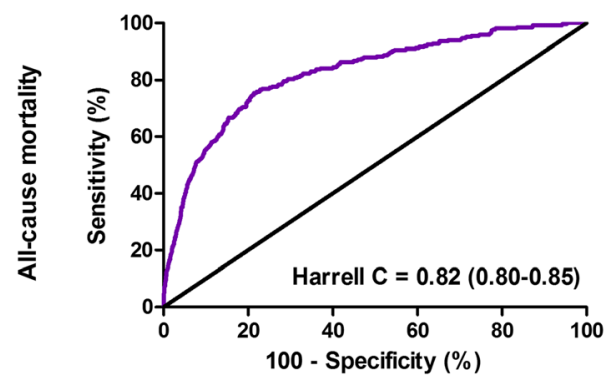

B

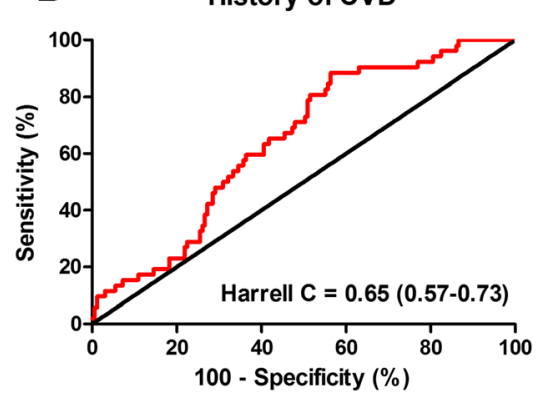

D

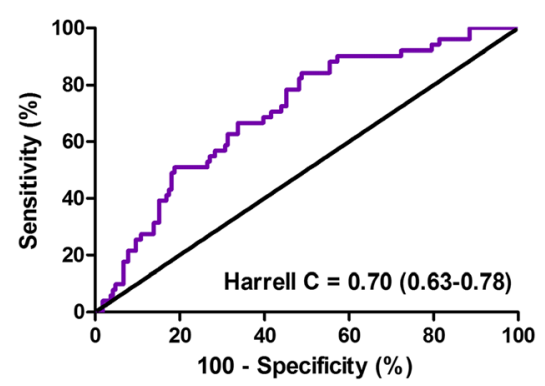

Fig. 5 Receiver operating characteristic (ROC) curves with associated Harrell C indices (95\% confidence interval [CI]) of Cox proportional hazards regression models in subjects with and without a history of cardiovascular disease (CVD). $\mathbf{a}$, b Discriminative ability of Cox regression models for the association between protein-adjusted serum-free thiols and cardiovascular events in both subgroups. $\mathbf{c}$, $\mathbf{d}$ Discriminative ability of Cox regression models for the association between protein-adjusted serum free thiols and all-cause mortality in both subgroups. CVD,

cardiovascular disease

found to be more negatively associated with CV events in subgroups characterized by the absence of obesity, hypertension, and diabetes. Conversely, free thiols were more negatively associated in subjects with albuminuria. Although we cannot clearly explain these findings, a possible explanation could be that albuminuria reflects ongoing endothelial damage that leads to a stronger prediction, whereas obesity and hypertension are merely risk factors and their presence does not significantly contribute to the predictive capacity. However, free thiols were more negatively associated with $\mathrm{CV}$ events in subjects without diabetes. Although it could be stated that subjects with diabetes might also show generalized manifest damage, the majority of these diabetic subjects had a relatively short disease duration. Therefore, we deem it unlikely that these subjects already had diabetes-induced microvascular complications. However, the value of free thiols in these specific subgroups requires further study as subgroup sizes were relatively unbalanced.

Free thiols (R-SH, sulfhydryl groups) are more accurately reflecting the systemic in vivo redox status as compared to many other individual oxidant or antioxidant factors and their derivatives [5]. In the extracellular environment, thiols are an integral part of a complex and dynamic redox signaling network and possess potent antioxidant activity based on their ability to rapidly scavenge circulating reactive species [18]. Extracellular thiols are predominantly embedded in plasma proteins, whereas a smaller percentage is represented by low-molecular-weight (LMW) thiols, such as cysteine, homocysteine, and glutathione (GSH) [37]. Blood proteins harbor the largest amount of redox-active thiol groups. In this respect, albumin is quantitatively the most important molecule within this pool (based on the existence of its single free cysteine residue, $\mathrm{Cys}^{34}$ ), given its abundance and because it is capable of transporting LMW thiols [18]. Collectively, systemic thiols circulate in both reduced and oxidized forms and, given their rapid and potent antioxidant activity, have a major impact on the net extracellular redox equilibrium. Therefore, measuring systemic free thiol availability may constitute a simple, robust, and easily reproducible method to assess the level of systemic oxidative stress in humans. Since proteins largely determine the total amount of measurable free thiol groups, adjusting these to circulating total protein or albumin levels can be regarded as an indirect approach to more precisely reflect the total serum free thiol pool $[7,8,10,11,38]$.

Due to the fact that free thiols are known to be receptive to therapeutic modulation, our results shed light on the potential development of therapeutic interventions. For instance, several thiol-based antioxidants, such as $\mathrm{N}$ acetylcysteine (NAC) or glutathione (GSH), have been investigated for their potential to restore extracellular 
thiol homeostasis in both human and animal studies [39]. Administration of these substances may increase the extracellular reducing capacity, though these potential effects should be carefully examined since they may disturb the diverse physiological functions of reactive species [40].

Strengths of the present study include the size and extensive clinical characterization of our study cohort and the long follow-up duration. However, several limitations also have to be taken into account. For instance, the observational nature of the study does not allow us to infer causality between serum levels of free thiols and CV events and all-cause mortality. In addition, given the fact that the majority of patients were Caucasians, the generalizability of our results in subjects with other ethnicities remains unknown. Likewise, the current study was carried out in the northern parts of the Netherlands; therefore, the generalizability of our results could be debated. Moreover, many arrows are absent in our directed acyclic graph (DAG) for which arguments for their presence could be made (for example, socio-economic status and BMI, socio-economic status and smoking, alcohol and BMI, smoking and BMI) [41-44]. However, adding these arrows as direct effects in Additional file 1: Figure S1 (scenario 3) would not have altered the set of confounders necessary to correct for in our analysis. In that sense, our analysis is robust to most of the underlying causal assumptions made.

\section{Conclusion}

In conclusion, we show that protein-adjusted serum free thiol levels are significantly predictive of the risk of $\mathrm{CV}$ events and all-cause mortality in the general population. Although free thiols might harbor great potential as an easily measurable biomarker in the current primary and secondary CVD prevention strategies, future prospective studies will be required to further elucidate the clinical relevance of serum free thiol levels in this context.

\section{Supplementary information}

Supplementary information accompanies this paper at https://doi.org/10. 1186/s12916-020-01587-w.

Additional file 1: Figure S1. Directed Acyclic Graph (DAG) showing the causal paths which were hypothesized to be involved in the relationship between systemic oxidative stress and the risk of cardiovascular events and all-cause mortality. Arrows depict hypothesized causal (direct) effects between variables, whereas absence of an arrow between two variables represents the assumption of no such direct effect.

Additional file 2: Figure S2 (A-B). Protein-adjusted serum free thiol concentrations $(\mu \mathrm{mol} / \mathrm{g})$ show no deviances from linear associations with either (A) the risk for CV events or (B) all-cause mortality. Estimated associations were derived from the adjusted Cox proportional hazards regression analysis (model 3) based on restricted cubic splines with three knots. $P$-values for non-linearity were $P=0.258$ for $C V$-events and $P=0.642$ for all-cause mortality. The median of protein-adjusted serum free thiol concentrations was taken as a reference standard $(5.07 \mathrm{\mu mol} / \mathrm{g}$ of protein). Gray-shaded areas represent 95\% confidence intervals.

\section{Acknowledgements}

All authors would like to thank the participants of the PREVEND study.

\section{Authors' contributions}

SJLB and RTG created the concept and design of the study. AEA and ARB performed the statistical analyses. HVG, DJM, SJLB, and RTG supervised the study. All authors were involved in the analysis and interpretation of the data and contributed to the drafting of the manuscript and critical revision for important intellectual content. All authors gave final approval for the article to be published.

\section{Funding}

The Prevention of REnal and Vascular End-stage Disease (PREVEND) study has been made possible by grants from the Dutch Kidney Foundation (by grant E.013). The funders of the work had no role in the conception, execution, or analysis of the research and had no role in drafting the manuscript.

\section{Availability of data and materials}

The datasets used and analyzed during the current study are available from the corresponding author on reasonable request.

\section{Ethics approval and consent to participate}

This study was approved by the Institutional Review Board (IRB) (full name in Dutch: "Medisch Ethische Toetsingscommissie" [METc]) of the UMCG. All study participants provided written informed consent. The study was conducted in accordance with the principles of the Declaration of Helsinki (2013)

\section{Consent for publication}

Not applicable.

\section{Competing interests}

The authors declare that they have no competing interests.

\section{Author details}

'Division of Vascular Medicine, Department of Internal Medicine, University of Groningen - University Medical Center Groningen, Groningen, the Netherlands. ${ }^{2}$ Department of Gastroenterology and Hepatology, University of Groningen - University Medical Center Groningen, Groningen, the

Netherlands. ${ }^{3}$ Division of Nephrology, Department of Internal Medicine, University of Groningen - University Medical Center Groningen, Groningen, the Netherlands. ${ }^{4}$ Department of Pathology and Medical Biology, Section Pathology, University of Groningen - University Medical Center Groningen, Groningen, the Netherlands. ${ }^{5}$ Department of Epidemiology, University of Groningen - University Medical Center Groningen, Groningen, the Netherlands. ${ }^{6}$ Division of Endocrinology, Department of Internal Medicine, University of Groningen - University Medical Center Groningen, Groningen, the Netherlands. ${ }^{7}$ Institute for Physiology and Pathophysiology, Johannes Kepler University Linz, Linz, Austria.

Received: 11 February 2020 Accepted: 6 April 2020

Published online: 27 May 2020

\section{References}

1. Lozano R, Naghavi M, Foreman K, Lim S, Shibuya K, Aboyans V, et al. Global and regional mortality from 235 causes of death for 20 age groups in 1990 and 2010: a systematic analysis for the Global Burden of Disease Study 2010. Lancet. 2012;380:2095-128.

2. Wang H, Naghavi M, Allen C, Barber RM, Carter A, Casey DC, et al. Global, regional, and national life expectancy, all-cause mortality, and cause-specific mortality for 249 causes of death, 1980-2015: a systematic analysis for the Global Burden of Disease Study 2015. Lancet. 2016;388:1459-544.

3. D'Agostino RB, Pencina MJ, Massaro JM, Coady S. Cardiovascular disease risk assessment: insights from Framingham. Glob Heart. 2013;8:11-23.

4. Banne AF, Amiri A, Pero RW. Reduced level of serum thiols in patients with a diagnosis of active disease. J Anti Aging Med. 2003;6:327-34. 
5. Cortese-Krott MM, Koning A, Kuhnle GGC, Nagy P, Bianco CL, Pasch A, et al. The reactive species interactome: evolutionary emergence, biological significance, and opportunities for redox metabolomics and personalized medicine. Antioxid Redox Signal. 2017;27(10):684-712.

6. Turrens JF. Mitochondrial formation of reactive oxygen species. J Physiol. 2003:552:335-44.

7. Koning AM, Meijers WC, Pasch A, Leuvenink HGD, Frenay ARS, Dekker MM et al. Serum free thiols in chronic heart failure. Pharmacol Res. 2016;111: 452-8.

8. Bourgonje AR, von Martels JZH, Bulthuis MLC, van Londen M, Faber KN, Dijkstra G, et al. Crohn's disease in clinical remission is marked by systemic oxidative stress. Front Physiol. 2019;10:2-7.

9. Liguori I, Russo G, Curcio F, Bulli G, Aran L, Della-Morte D, et al. Oxidative stress, aging, and diseases. Clin Interv Aging. 2018;13:757-72.

10. Abdulle AE, van Roon AM, Smit AJ, Pasch A, van Meurs M, Bootsma $\mathrm{H}$, et al. Rapid free thiol rebound is a physiological response following cold-induced vasoconstriction in healthy humans, primary Raynaud and systemic sclerosis. Physiol Rep. 2019;7(6):e14017.

11. Frenay ARS, de Borst MH, Bachtler M, Tschopp N, Keyzer CA, van den Berg

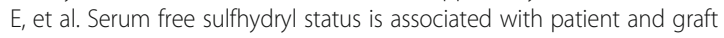
survival in renal transplant recipients. Free Radic Biol Med. 2016;99:345-51.

12. Go YM, Jones DP. Cysteine/cystine redox signaling in cardiovascular disease. Free Radic Biol Med. 2011:50:495-509.

13. Hillege HL, Janssen WMT, Bak AAA, Diercks GFH, Grobbee DE, Crijns HJGM, et al. Microalbuminuria is common, also in a nondiabetic, nonhypertensive population, and an independent indicator of cardiovascular risk factors and cardiovascular morbidity. J Intern Med. 2001;249:519-26.

14. Kieneker LM, Gansevoort RT, De Boer RA, Brouwers FP, Feskens EJM, Geleijnse JM, et al. Urinary potassium excretion and risk of cardiovascular events. Am J Clin Nutr. 2016;103:1204-12.

15. Grubb A, Blirup-Jensen S, Lindström V, Schmidt C, Althaus H, Zegers I. First certified reference material for cystatin $C$ in human serum ERM-DA471/IFCC. Clin Chem Lab Med. 2010;48:1619-21.

16. Ellman GL. Tissue sulfhydryl groups. Arch Biochem Biophys. 1959;82:70-7.

17. Hu M-L, Louie S, Cross CE, Motchnik P, Halliwell B. Antioxidant protection against hypochlorous acid in human plasma. J Lab Clin Med. 1993;121:257-62.

18. Turell $L$, Radi R, Alvarez B. The thiol pool in human plasma: the central contribution of albumin to redox processes. Free Radic Biol Med. 2013;65: $244-53$

19. Inker LA, Schmid CH, Tighiouart H, Eckfeldt JH, Feldman HI, Greene T, et al. Estimating glomerular filtration rate from serum creatinine and cystatin C. N Engl J Med. 2012:367(1):20-9.

20. WHO. International Statistical Classification of Diseases and Related Health Problems - 10th revision. World Heal Organ. 2011;2.

21. Kip KE, Hollabaugh K, Marroquin OC, Williams DO. The problem with composite end points in cardiovascular studies. The story of major adverse cardiac events and percutaneous coronary intervention. J Am Coll Cardiol. 2008:51:701-7.

22. Pearl J. Causality, models, reasoning, and inference. New York: Cambridge University Press; 2000.

23. La Bastide-Van Gemert S, van den Heuvel E. Exploring causal hypotheses: breaking with long-standing research traditions. Dev Med Child Neurol. 2013;55:975-6.

24. Lederer DJ, Bell SC, Branson RD, Chalmers JD, Marshall R, Maslove DM, et al. Control of confounding and reporting of results in causal inference studies. Guidance for authors from editors of respiratory, sleep, and critical care journals. Ann Am Thorac Soc. 2019;16:22-8.

25. Erhardt L. Cigarette smoking: an undertreated risk factor for cardiovascular disease. Atherosclerosis. 2009:205:23-32.

26. Singh-Manoux A, Shipley MJ, Bell JA, Canonico M, Elbaz A, Kivimaki M. Association between inflammatory biomarkers and all-cause, cardiovascular and cancer-related mortality. CMAJ. 2017;189:E384-90.

27. Wonisch W, Falk A, Sundl I, Winklhofer-Roob BM, Lindschinger M. Oxidative stress increases continuously with $\mathrm{BMl}$ and age with unfavourable profiles in males. Aging Male. 2012;15:159-65.

28. Münzel T, Gori T, Bruno RM, Taddei S. Is oxidative stress a therapeutic target in cardiovascular disease? Eur Heart J. 2010;31:2741-8.

29. Tzoulaki I, Elliott P, Kontis V, Ezzati M. Worldwide exposures to cardiovascular risk factors and associated health effects: current knowledge and data gaps. Circulation. 2016;133:2314-33.
30. Pencina MJ, D'Agostino RB, Larson MG, Massaro JM, Vasan RS. Predicting the 30-year risk of cardiovascular disease: the Framingham Heart Study. Circulation. 2009;119:3078-84.

31. Liao J, Muniz-Terrera G, Scholes S, Hao Y, Chen YM. Lifestyle index for mortality prediction using multiple ageing cohorts in the USA, UK and Europe. Sci Rep. 2018;8:6644

32. Salzano S, Checconi P, Hanschmann EM, Lillig CH, Bowler LD, Chan P, et al. Linkage of inflammation and oxidative stress via release of glutathionylated peroxiredoxin-2, which acts as a danger signal. Proc Natl Acad Sci U S A. 2014;111:12157-62.

33. Cervantes Gracia K, Llanas-Cornejo D, Husi H. CVD and oxidative stress. J Clin Med. 2017;6:E22.

34. Kundi H, Ates I, Kiziltunc E, Cetin M, Cicekcioglu H, Neselioglu S, et al. A novel oxidative stress marker in acute myocardial infarction; thiol/disulphide homeostasis. Am J Emerg Med. 2015;33:1567-71.

35. Belch JJ, Bridges AB, Scott N, Chopra M. Oxygen free radicals and congestive heart failure. Br Heart J. 1991;65:245-8.

36. Mangoni AA, Zinellu A, Carru C, Attia JR, McEvoy M. Serum thiols and cardiovascular risk scores: a combined assessment of transsulfuration pathway components and substrate/product ratios. J Transl Med. 2013;11: 99.

37. Hortin GL, Sviridov D, Anderson NL. High-abundance polypeptides of the human plasma proteome comprising the top 4 logs of polypeptide abundance. Clin Chem. 2008:54:1608-16.

38. Anraku M, Chuang VTG, Maruyama T, Otagiri M. Redox properties of serum albumin. Biochim Biophys Acta - Gen Subj. 1830;2013:5465-72.

39. Deneke SM. Thiol-based antioxidants. Curr Top Cell Regul. 2000:151-80.

40. Atkuri KR, Mantovani JJ, Herzenberg LA, Herzenberg LA. N-acetylcysteine-a safe antidote for cysteine/glutathione deficiency. Curr Opin Pharmacol. 2007:7:355-9.

41. Abreu S, Santos R, Moreira C, Santos PC, Mota J, Moreira P. Food consumption, physical activity and socio-economic status related to BMI, waist circumference and waist-to-height ratio in adolescents. Public Health Nutr. 2014;17:1834-49

42. Meijer E, Gebhardt WA, Van Laar C, Kawous R, Beijk SCAM. Socio-economic status in relation to smoking: the role of (expected and desired) social support and quitter identity. Soc Sci Med. 2016:162:41-9.

43. Liao C, Gao W, Cao W, Lv J, Yu C, Wang S, et al. The association of cigarette smoking and alcohol drinking with body mass index: a cross-sectional, population-based study among Chinese adult male twins. BMC Public Health. 2016;31(16):311.

44. Wang P, Abdin E, Sambasivam R, Chong SA, Vaingankar JA, Subramaniam M. Smoking and socio-demographic correlates of BMI. BMC Public Health. 2016;16:500

\section{Publisher's Note}

Springer Nature remains neutral with regard to jurisdictional claims in published maps and institutional affiliations.

Ready to submit your research? Choose BMC and benefit from:

- fast, convenient online submission

- thorough peer review by experienced researchers in your field

- rapid publication on acceptance

- support for research data, including large and complex data types

- gold Open Access which fosters wider collaboration and increased citations

- maximum visibility for your research: over $100 \mathrm{M}$ website views per year

At $\mathrm{BMC}$, research is always in progress.

Learn more biomedcentral.com/submission 